\title{
Análise epistemológica dos conhecimentos científicos sobre Toxocara sp. com ênfase na infecção humana
}

\author{
Epistemological analysis of the scientific knowledge about \\ Toxocara sp. with the emphasis on human infection
}

Silvia Rafaelli Marques ${ }^{1}$

Leucio Câmara Alves ${ }^{1}$

Maria Aparecida da Glória Faustino ${ }^{1}$

${ }^{1}$ Departamento de

Abstract The scope of this article was to analyze the evolution of knowledge about Toxocara sp. from a zoonotic point of view by means of scientific data published between the years 1996 and 2015 using epistemology as a tool. The epistemological analysis of the evolution of knowledge on the zoonotic aspect of Toxocara sp. in Brazil revealed that, in general, little new information was added which had, over the period studied, slow and discontinuous absorption, and was not incorporated in the social reality from the standpoint of public health.

Key words Human toxocariasis, Way of thinking, Visceral larva migrans
Resumo Este artigo objetivou analisar a evolução do conhecimento sobre Toxocara sp. em seu aspecto zoonótico por meio de dados científicos entre os anos de 1996 a 2015 utilizando a epistemologia como ferramenta. A análise epistemológica da evolução do conhecimento sobre o aspecto zoonótico de Toxocara sp. no Brasil demonstrou que, de uma maneira geral, foram agregadas poucas novas informações as quais tiveram, ao longo do período estudado, absorção lenta, descontínua, e não foram inseridas na realidade social do ponto de vista de Saúde Pública.

Palavras-chave Toxocaríase humana, Estilo de pensamento, Larva migrans visceral 


\section{Introdução}

A Epistemologia é um conjunto de conhecimentos que tem por objetivo o conhecimento científico, visando explicar o seu condicionamento seja ele técnico, histórico, ou social e assim sistematizar as suas relações, esclarecendo os seus vínculos e avaliar os seus resultados e aplicações ${ }^{1,2}$.

As investigações epistemológicas podem desenvolver um melhor resultado em processos de elaboração de conhecimento podendo auxiliar no combate das parasitoses destacando as helmintoses. A busca de dados mais detalhados sobre o conhecimento teórico, histórico e técnico da parasitologia helmíntica pode ser baseada em dados investigativos no ambiente onde ela se desenvolve com o uso da epistemologia como ferramenta ${ }^{3,4}$.

A investigação e avaliação de acontecimentos já estabelecidos e sobre os quais são conservadas as opiniões e fazem parte da vida prática seriam apropriados para reflexões epistemológicas na área da biologia e da saúde ${ }^{5,6}$. Neste contexto, as helmintoses zoonóticas podem ser um objeto de estudo epistemológico (estilo de pensamento), possibilitando notar as modificações e/ou modernizações e assim proporcionando outras opiniões para efetuar ou elaborar estudos mais eficazes no diagnóstico, tratamento e prevenção das mesmas, que apesar de serem conhecidas e estudadas há bastante tempo são atualmente importantes para a Saúde Pública em muitos países, incluindo o Brasil ${ }^{7-9}$.

Um dos helmintos zoonóticos mais conhecidos é Toxocara sp. que compreende 21 espécies, tendo as espécies Toxocara canis e Toxocara cati como as mais implicadas na síndrome da Larva Migrans Visceral (LMV) ${ }^{9,10}$. O hospedeiro definitivo são animais domésticos, nos quais o parasito vive como adulto dentro do lúmen do intestino delgado $^{11}$. A Larva Migrans Visceral foi descrita em 1952 como forma sistêmica grave, caracterizada por eosinofilia elevada, hepatoesplenomegalia, febre, dentre outras complicações e ainda observados $^{12-14}$. São zoonoses parasitárias negligenciadas, causadas por ingestão de ovos de Toxocara canis e de Toxocara cati. Quando os ovos contendo larvas infectantes são ingeridos por seres humanos, as larvas tornam-se livres no intestino, mas não conseguem se desenvolver até a forma adulta. Ao invés disso, atravessam a parede intestinal e estabelecem-se nos tecidos, podendo invadir órgãos como o fígado, pulmões, olhos ou cérebro $^{15}$.
O objetivo deste artigo foi analisar a evolução do conhecimento sobre Toxocara sp. em seu aspecto zoonótico por meio de dados científicos entre os anos de 1996 a 2015 utilizando a epistemologia como ferramenta.

\section{Método}

Artigos e textos foram obtidos por meio das fontes de pesquisa científicas Pubmed, Medline, SciELO, Lilacs, textos publicados em sites relevantes como: Organização Mundial da Saúde (OMS), Organização Pan-Americana da Saúde (OPAS), Fundação Instituto Oswaldo Cruz (Fiocruz), Ministério da Saúde e bancos de dissertações e teses, registrados de 1996 a 2015. Como estratégia de busca foram utilizados os termos helmintoses, helmintoses zoonóticas, geohelmintos, Toxocara sp. e Larva migrans visceral, em inglês e português, incluindo-se apenas estudos realizados no Brasil cujo foco era Toxocara sp. e se reportavam ao seu caráter zoonótico. Foram excluídos estudos realizados fora do Brasil e aqueles em que estavam envolvidos protozoários.

A análise epistemológica foi realizada segundo Fleck ${ }^{5}$. Os artigos e textos foram organizados inicialmente em: revisão, artigo original, relato de casos, textos de sites, dissertação e tese, em seguida, separados nas classes: produções com ênfase na epidemiologia e produções com ênfase na educação em saúde. Em determinados casos, foi necessário utilizar alguns artigos não incluídos na análise (conforme os critérios de inclusão e exclusão acima definidos) para dar maior consistência à discussão.

\section{Resultados e Discussão}

A partir dos descritores foram encontrados 7.043 textos científicos, sendo pré-selecionados 669 referentes a helmintoses zoonóticas, dos quais 79 eram sobre helmintoses zoonóticas envolvendo animais de estimação e 48 destes se reportavam a Toxacara sp. e seu caráter zoonótico, sendo, então analisados.

Quanto ao tipo de texto científico, 93,68\% (41/48) eram artigos originais, dois (2,53\%) artigos de revisão, duas $(2,53 \%)$ teses e uma dissertação $(1,26 \%)$, todos textos relacionados com epidemiologia, não se encontrando nenhuma alusão à educação em saúde.

Os resultados alcançados pela análise epistemológica foram separados em tópicos. 


\section{Estilos de pensamento evidenciados}

Uma análise a partir deste ponto de vista requer inicialmente uma reflexão sobre os conceitos de Saúde Pública. A definição de Saúde Pública está em constante evolução ao observar a literatura científica, contudo, de acordo com Lechopier $^{16}$ foi adotada uma definição possivelmente consensual, proposta pelo Conselho Nuffield de Bioética, em 2007, segundo a qual Saúde Pública corresponde à "ciência e a arte de prevenir a doença, prolongar a vida e promover a saúde por meio de esforços organizados da sociedade". Revendo artigos anteriores à citação de 2015, conclui-se que esta definição foi postulada em 1988, apresentando-se como um resumo de uma versão proposta em $1920^{17-19}$.

Muitas são as expressões aplicadas às doenças do ponto de vista de seu impacto na Saúde Pública: "doenças de interesse para a Saúde Pública", "problema de Saúde Pública", "doença de importância para a Saúde Pública”. A expressão "problema de Saúde Pública" parece ser a mais comumente aplicada e foi revisada por Costa e Victora $^{20}$ em textos de medicina preventiva e Saúde Pública apresentada abaixo (Quadro 1).

Costa e Victora ${ }^{20}$ acrescentam, ainda, o potencial epidêmico da enfermidade. Contudo consideram que, para ser enquadrada como problema para a Saúde Pública, não há necessidade de a enfermidade atender a todos os critérios listados, além de não se poder igualar o nível de importância das mesmas para a sociedade.

Com base nas definições acima, as reflexões epistemológicas sobre Toxocara sp. conduzem aos seguintes estilos de pensamento: importância para Saúde Pública, problema de Saúde Pública. Dos 48 textos científicos analisados 33,34\% $(16 / 48)^{21-36}$ referem-se a problema de Saúde
Pública; 29,16\% (14/48) enfatizam sua importância em Saúde Pública ${ }^{10,42-55}, 37,50 \%$ (18/48) reportam-se simplesmente ao caráter zoonótico $^{13,15,56-71}$, sem levar em consideração os estilos de pensamento anteriormente citados. Convém informar que todos os 48 textos científicos retratam o caráter zoonótico. Pode-se destacar, ainda, que Gallina et al. ${ }^{48}$ e Mata-Santos et al. ${ }^{15}$ mencionam a toxocaríase como doença subestimada.

Quanto à questão de a toxocaríase ser um problema de Saúde Pública não se observou evolução do conhecimento, o que pode ter ocorrido, talvez, devido a não haver uma definição consensual para o termo "problema de Saúde Pública", sendo, então a expressão utilizada pelo autor para dar maior ênfase ao seu tema de estudo como verificado por Costa e Victora ${ }^{20}$.

Com relação à evolução do conhecimento sobre a importância de Toxocara sp. para a Saúde Pública também não foi acrescentada nenhuma nova informação no decorrer dos anos nos artigos estudados, permanecendo sua relevância relacionada ao fato de causar a Larva migrans visceral em humanos, os danos provocados à saúde humana e animal, além da contaminação do solo como potencial fonte de infecção humana.

Embora tanto o termo "importância para a Saúde Pública" quanto "problema de Saúde Pública" tenham sido aplicados para as enfermidades causadas por Toxocara sp., quando se consideram os critérios citados por Costa e Victora ${ }^{20}$, chega-se a conclusão de que Toxocara sp. não se constitui, realmente, um problema de Saúde Pública diante dos objetivos dos trabalhos pesquisados. Por outro lado, a questão de ser importante para a Saúde Pública, pode ser aventada, mesmo que em pequena proporção, levando-se em consideração o impacto clínico sobre o indivíduo acometido.

Quadro 1. Critérios para definição de problema de saúde pública segundo Costa e Victora ${ }^{20}$.

\begin{tabular}{|c|c|}
\hline Autores & Critérios definidos para definir problema de saúde pública \\
\hline Leavell $(1976)^{37}$ & \multirow[t]{2}{*}{ Natureza, Extensão, severidade e significância } \\
\hline Clark e Macmahon, $(1967)^{38}$ & \\
\hline Morley $(1980)^{39}$ & Interesse da comunidade, prevalência, gravidade e possibilidade de controle. \\
\hline Fowler e Austoker (1997) ${ }^{40}$ & $\begin{array}{l}\text { Impacto no indivíduo em termos de anos potenciais de vida perdidos, extensão } \\
\text { de incapacidade, dor e desconforto, custo do tratamento, e impacto na família } \\
\text { do indivíduo } \\
\text { Impacto na sociedade - mortalidade, morbidade e custos do tratamento para a } \\
\text { sociedade. }\end{array}$ \\
\hline Daly et al. $(2002)^{41}$ & $\begin{array}{l}\text { Prevalência da condição, impacto da condição no nível individual, seu impacto } \\
\text { na sociedade (do ponto de vista econômico) e se a condição pode ser prevenida } \\
\text { ou se existe um tratamento efetivo disponível. }\end{array}$ \\
\hline
\end{tabular}




\section{Toxocara sp. como agente de doença no homem}

Toxocara sp. é conhecido como agente etiológico da Larva migrans visceral desde $1952^{13}$. A partir de então vem sendo alvo de inúmeras pesquisas, reunindo aspectos que incluem: ser a causa da síndrome da Larva migrans visceral que afeta a saúde dos seres humanos causando lesões, às vezes, irreparáveis ${ }^{11,34,72-74}$; causar doença nos animais domésticos ${ }^{11}$; ser uma zoonose; contaminar o solo ${ }^{11,15,24,50,57,59,74}$.

A disseminação do conhecimento científico dos eventos sobre as enfermidades causadas por Toxocara sp. são encontrados nos artigos mais antigos como Beaver et al. ${ }^{12}$ e também nos atuais como Stalliviere et al. ${ }^{29}$ e Onuma et al..$^{54}$.

Apesar de todos estes fatores expressivos, as síndromes e doenças causadas pelo Toxocara sp. no homem são subdiagnosticadas o que favorece a negligenciação de sua prevalência e incidência atuais ${ }^{15,49,52}$.

Estudos demonstram a prevalência sorológica da toxocaríase no Brasil variando de 2,2 a $54,8 \% \%^{10,22,24,32,34,50,51,57,59,64,65,72,73,75,76}$. As diferenças entre as taxas verificadas nos artigos citados pode ser justificada pela faixa etária dos indivíduos incluídos no estudo. Destacam-se maiores taxas de prevalência entre crianças $\mathrm{s}^{34,51}$ pelo maior contato com cães, hábitos de geofagia e onicofagia ${ }^{34}$

Dentro do período analisado no presente estudo, apenas um artigo foi encontrado, detectando taxa anual de incidência de $17,9 \%$ para infecção por Toxocara sp. em moradores da periferia de Campinas $-\mathrm{SP}^{74}$. A ausência de dados sobre a taxa de incidência para Toxocara sp. tanto no País como em outras regiões deve-se ao fato de que quase todas as investigações de campo são concebidas como estudos transversais, considerandose apenas um momento da história natural da infecção ${ }^{74}$.

A contaminação do solo por ovos de Toxocara sp. vem sendo referenciada desde $1984^{77}$, e prossegue até anos mais recentes, como 2014, sem alterações no conhecimento já obtido ${ }^{27,28,33,42,51,54,57,78-81}$ e sinaliza para uma maior atenção com os animais domésticos em áreas públicas e apontam animais errantes como possíveis auxiliares na permanência e disseminação deste helminto ${ }^{34,42,51,54}$.

A infecção humana por meio da ingestão de alimentos contaminados com ovos embrionados $^{22}$ deve-se a maus hábitos de higiene como a manipulação de alimentos com as mãos sujas $^{22,34}$ após contato com animal parasitado ou solo contaminado ${ }^{22,34,82}$. Embora os textos citados refiram-se a alimentos sem citar a natureza dos mesmos, a contaminação de hortaliças por ovos de Toxocara sp. é citada em diferentes estudos ${ }^{81-83}$. A contaminação de carne crua ou mau passada é reportada por Andrade ${ }^{22}$ e Santarém et al..$^{32}$, não havendo relatos desta via de infecção em estudos brasileiros.

A infecção por alimentos contaminados constitui-se em informação acrescentada ao longo dos anos de estudo, portanto mais recente que a contaminação do solo, especialmente a carne crua ou mau passada. Andrade ${ }^{22}$ expõe que certos hábitos culturais também podem representar fator de risco para infecção por Toxocara sp., entre estes o hábito de comer carnes e vísceras cruas observado em certas populações. Nesta reflexão epistemológica demonstra-se claramente que o conhecimento é vinculado e depende de fatores socioculturais, exercendo influências sobre a realidade social ${ }^{5,6}$. Assim. estudos realizados em nosso meio não incluem esta linha de investigação pelo fato de o consumo de carne e vísceras cruas não serem hábitos comuns em nossa sociedade.

\section{Patogênese}

Após a ingestão dos ovos larvados e eclosão, no intestino delgado as larvas ganham a circulação pela via hepática migrando para os diferentes órgãos, induzindo alterações da diversas naturezas, como aumento considerável de eosinófilos, aumento das globulinas séricas com altos títulos de IgG e IgM, hepatomegalia, hepatoesplenomegalia, nódulos nos órgãos e derrame pleural, nódulos pulmonares ou hepáticos e pneumonia $^{10,12,13,31,75}$, aumento da IgE total, alta susceptibilidade a asma, leucocitose e anemia ${ }^{14,25,61}$, hemorragia, necrose e inflamação eosinofilica ${ }^{11,35}$. Há relatos de pleocitose e mielite, alterações neurológicas que podem estar relacionadas com vasculite cerebral, e convulsões ${ }^{10,14,32}$.

\section{Sinais clínicos}

Muitas das infecções de Toxocara sp. são assintomáticas ${ }^{12,22,34}$ contudo pode ocorrer a Toxocaríase sistêmica que possui uma variabilidade de sintomas e sinais clínicos tais como febre, tosse, sintomas gripais, fadiga, problemas gastrintestinais, enxaqueca, dor abdominal, dor articular, dor de cabeça, alterações respiratórias, chiado persistente, erupções cutâneas, pápulas eritematosas e urticária ${ }^{10,12,22,24,75,76}$.

Andrade ${ }^{22}$ já chamava a atenção para o fato de a toxocaríase em humanos apresentar varian- 
tes clínicas apesar de o termo toxocaríase ser, com muita frequência, utilizado na literatura como sinônimo de Larva migrans visceral (expressão clínica mais conhecida da toxocaríase) devido à destacada importância de T. canis na epidemiologia desta síndrome. Em sua revisão, o autor faz referência à Toxocaríase ocular para qual consta citação de 1953 e toxocaríase oculta relatada em 1987.

No presente estudo, a maioria dos textos pesquisados referem-se à toxocaríase utilizando o termo Larva migrans visceral, mesmo os mais recentes $^{13,51}$. No entanto, tem-se um histórico bastante claro da classificação da toxocaríase humana ${ }^{11}$ baseada em artigos estrangeiros: Toxocaríase assintomática, Toxocaríase oculta, Larva migrans visceral, Larva migrans ocular e Larva migrans neurológica.

Assim, observa-se que a divulgação de novas informações sobre formas clínicas da toxocaríase humana no Brasil não acompanhou suficientemente a literatura científica, pois termos novos relacionados à classificação clínica não foram incorporados aos artigos publicados no Brasil pela maioria dos autores.

A despeito da situação acima colocada, pela visão epistemológica, o conhecimento científico sobre sinais clínicos da toxocaríase evoluiu a partir de ideias iniciais, surgidas no passado, relativas a este assunto, as quais foram pouco a pouco modificadas e sofrendo reinterpretações de acordo com o pensamento em evidência, mas continuaram existindo, apesar das modificações ${ }^{5,6}$

\section{Diagnóstico}

As síndromes de toxocaríase humana são de difícil diagnóstico pois, em geral, além do fato de serem confundidas com outras enfermidades, as lesões patológicas são muitas vezes não relacionadas com a doença $a^{10,13,22,32}$.

Embora o diagnóstico presuntivo se baseie em um conjunto de dados clínicos, laboratoriais e epidemiológicos ${ }^{22,65}$, a falta de diagnóstico laboratorial e sintomas específicos faz com que o diagnóstico da toxocaríase humana seja raramente realizado, levando-a a ser considerada uma doença negligenciada nos serviços de saúde ${ }^{13,22,51,57,75}$.

O diagnóstico direto da infecção humana é uma tarefa difícil, por não haver desenvolvimento do parasito até a maturidade no organismo humano. Porém pode ser realizado por meio de biópsia para detectar a presença da larva nos tecidos ${ }^{11,23}$. Este procedimento raramente é recomendado por expor o paciente a riscos além de o exame negativo não constituir resultado conclusivo $^{10,22}$. Portanto os métodos indiretos por detecção de elevados níveis de anticorpos anti-T. canis em fluidos biológicos tornaram-se os meios de diagnósticos mais adequados ${ }^{22,84}$. O diagnóstico sorológico é realizado pelo teste imunoenzimático (ELISA) no qual são utilizados produtos antigênicos obtidos de cultura de larvas de T. canis - antígenos excretores-secretores (TES). Este método é amplamente usado nos inquéritos sorológicos em diferentes estudos no Brasil $25,35,48,51,52,58,60,66,74,77,85$, considerado de boa acurácia, possui limitação de ter reação cruzada com outros parasitos em países onde a infecção é prevalente. Como alternativa para minimizar esta desvantagem, normalmente, o soro é adsorvido com extratos de Ascaris suum antes dos testes para evitar a reação cruzada principalmente com Ascaris lumbricoides ${ }^{10,47,52,74,77,85}$.

Alguns kits para diagnóstico sorológico são comercialmente disponíveis, mas raramente utilizados no Brasil devido ao alto custo ${ }^{51}$ e, além disso, tem uso limitado em países tropicais por não determinarem prévia adsorção do soro com antígenos de parasitos que tenham reação cruzada com T. canis $^{65}$.

A imaginologia por meio da ressonância magnética pode ser especialmente útil no caso de Toxocaríase neurológica devido à inespecificidade dos sinais clínicos ${ }^{65}$. Cita-se também a ultrassonografia e tomografia computadorizada ${ }^{10}$. Não há relatos do seu uso no Brasil.

Técnicas moleculares como a Reação em Cadeia da Polimerase (PCR) não se encontram na literatura ora pesquisada, porém são citadas como futuras ferramentas para diagnóstico e prevenção ${ }^{33}$, podendo detectar DNA do parasito em tecidos ${ }^{66}$.

\section{Tratamento}

Quando diagnosticadas as síndromes, o tratamento varia de acordo com o conjunto de achados clínicos ou laboratoriais. Casos assintomáticos não entram nesse contexto ${ }^{10,14,22,33}$.

Andrade $^{22}$ propõe uma conduta relacionada à droga (tratamento anti-helmíntico visando debelar a infecção) e, uma conduta, ligada ao esquema de tratamento (tratamento sintomático objetivando reverter os sinais e sintomas). Deve-se consider a relação risco - benefício entre o potencial tóxico da droga e o efeito terapêutico esperado para a reversão do quadro clínico ${ }^{22,74}$.

Embora não exista um esquema de tratamento comprovadamente eficaz ${ }^{48}$, indicam-se, 
nos casos de Larva migrans visceral, drogas com propriedades larvicidas, como dietilcarbamazina, albendazol, mebendazol, levamisol, ivermectina, fenbendazol e oxfendazol, destacando-se o tiabendazol pela ação inibidora da migração tecídual das larvas ${ }^{10,14,22,33,48}$.

A conduta para casos de Larva migrans ocular deve avaliar a acuidade visual, gravidade da inflamação e reversibilidade da lesão ocular. O tratamento com anti-helmínticos é secundário, sendo utilizado no início da infecção e associado com a terapia de esteroides anti-inflamató$\operatorname{rios}^{14,22}$. O tratamento com dietilcabamazina e tiabendazol não é unanimemente aceito devido à possibilidade de morte das larvas e com isso aumento da reação inflamatória ${ }^{14}$.

Quanto ao tratamento da toxocaríase humana em indivíduos soropositivos assintomáticos, de um lado considera-se não haver indicação formal do ponto de vista epidemiológico devido ao diagnóstico ser baseado na sorologia sem comprovação parasitológica, por outro, justifica-se o tratamento devido à existência de risco de complicações futuras em indivíduos soropositivos ${ }^{22,48}$.

\section{Prevenção}

As medidas de controle das enfermidades causadas pelo Toxocara sp. são em maioria de fácil reprodução, incluindo ações de simples execução podendo ser realizadas pelo indivíduo até algumas outras mais complexas em sua operacionalidade. Podem ser evidenciadas três grupos de ações:

- As ações relacionadas aos animais de estimação que compreendem o controle da população canina, podendo ressaltar, para este grupo, os animais errantes e semi-domiciliados, o controle dos mesmos em áreas públicas e de lazer e o diagnóstico e tratamento ${ }^{10,14,15,33,35,48,49,54,61}$.

- A descontaminação do solo, envolvendo a remoção das fezes, em áreas adjacentes ao domicílio e piso dos canis sem frestas e $\operatorname{secos}^{54}$. Novas alternativas vem sendo buscadas, como a utilização de métodos que inviabilizem os ovos infectantes presentes nas fezes a exemplo do controle biológico por meio do fungo Pochonia chamidosporia, avaliado in vitro ${ }^{67}$.

- As atividades educativas direcionadas à população, com a participação das instituições governamentais que gerenciam programas de controle de doenças e seu entendimento sobre a necessidade de inclusão das medidas educativas em seu plano de intervenção junto às comunidades ${ }^{14,34,48,61,67}$. Citam-se a elaboração de futuros estudos e programas para a prevenção da infecção humana, orientação e conscientização dos profissionais de saúde, poderes públicos, educadores e clínicos veterinários sobre medidas educativas para reduzir os riscos de exposição a parasitos intestinais para seus clientes ${ }^{14,33,69}$. As pesquisas realizadas sobre prevenção da toxocaríase humana no Brasil mostram-se relativamente recentes, porém, delas se percebe que ainda há dificuldades no controle da doença. As formas de prevenção são indicadas, mas não são implementadas na prática. Não existem artigos que relatem a realização de intervenções para diminuir a prevalência da doença ou a realização de atividades de educação em saúde nos locais de ocorrência dos casos.

\section{Conclusões}

A análise epistemológica da evolução do conhecimento sobre o aspecto zoonótico de Toxocara sp. no Brasil demonstra que, de uma maneira geral, foram agregadas poucas novas informações as quais tiveram, ao longo do período estudado, absorção lenta, descontínua e não inseridas na realidade social do ponto de vista de Saúde Pública.

Não há uma definição clara da importância zoonótica de Toxocara sp. por não haver consenso sobre o termo "Problema de Saúde Pública" e pelo fato de as pesquisas realizadas sobre o toxocaríase humana não incluírem análises dos parâmetros apontados pela pesquisa científica como critérios para assim classificá-la.

Apesar da evolução do conhecimento sobre sinais clínicos do ponto de vista epistemológico, nota-se que este conhecimento não foi absorvido suficientemente, prevalecendo, ainda a utilização dos termos Larva migrans visceral e toxocaríase humana como sinônimos por muitos autores em detrimento de uma classificação clínica mais específica já existente.

Informações relacionadas ao diagnóstico apresentam novas opções metodológicas, as quais não são relatadas em trabalhos nacionais em relação ao seu uso na prática. Quanto ao tratamento e prevenção, o conhecimento existente vem sendo repassado ao longo dos anos sem profundas modificações.

Assim, as lacunas existentes sobre o conhecimento do tema precisam ser preenchidas de forma coerente pelos pesquisadores e adequadamente transferidas à comunidade científica, profissionais da área e poderes públicos para se 
estabelecer o diagnóstico de situação da Toxocaríase humana no Brasil visando a obtenção de propostas de intervenções mais favoráveis à saúde humana.

\section{Colaboradores}

SR Marques, LC Alves e MAG Faustino participaram diretamente no planejamento, execução ou análises deste estudo. Todos os autores deste artigo leram e aprovaram a versão final enviada.

\section{Referências}

1. Ferreira ABH. Miniaurélio Século XXI. $5^{\mathrm{a}}$ ed. Rio de Janeiro: Nova Fronteira; 2007.

2. Calvo MO, Torres PR, Peralta JL. La epistemología como propedéutica de la investigación sanitária. Gac Sanit 2011; 25(1):79-83.

3. Patz JA, Graczyk TK, Geller N, Vittor AY. Effects of environmental change on emerging parasitic diseases. Int J Parasitol 2000; 30(12-13):1395-1405.

4. Araújo A, Telles FSP. A parasitologia como exemplo da possibilidade de regras gerais para a ciência Parasitology as an example of the potential for general rules for Science. Fundação Oswaldo Cruz 2010; 17(1):211.

5. Fleck L. La génesis y el desarrollo de un hecho científico. Madrid: Alianza Editorial; 1986.

6. Pfuetzenreiter MR. Epistemologia de Ludwik Fleck Como referencial para a pesquisa nas ciências aplicadas. Episteme 2003; (16):111-135.

7. Katz N, Peixoto SV. Análise crítica da estimativa do número de portadores de esquistossomose mansoni no Brasil. Rev. Soc Bras Medic Tropic 2000; 33(3):303-308.

8. Favre TC, Pieri OS, Barbosa CS, Beck L. Avaliação das ações de controle da esquistossomose implementadas entre 1977 e 1996 na área endêmica de Pernambuco, Brasil. Rev Soc Bras Medic Tropic 2001; 34(6):569-576.

9. Uecker M, Copetti C, Poleze L, Flores V. Infecções parasitárias: diagnóstico imunológico de enteroparasitoses. Rev Bras Anal Clin 2007; 39(1):15-19.

10. Carvalho EA, Rocha RL. Toxocaríase: Larva migrans visceral em crianças e adolescentes. J Pediatriacs 2011; 87(2):100-110.

11. Despommier D. Toxocariasis: clinical aspects, epidemiology, medical ecology, and molecular aspects Clin Microbiol Rev, 2003; 16(2):265-272.

12. Beaver PC, Snyder H, Carrera G, Dent HA, Laffety GM Chronic eosinophilia due to visceral larva migrans: report of three cases. Pediatrics, 1952; 2(9):7-19.

13. Machado AB, Achkar MEE. Larva migrans visceral: um relato de caso. An Bras Dermatol 2003; 78(2):215-219.

14. Peruca LCB, Langoni H, Lucheis SB. Larva Migrans Visceral e Cutânea como Zoonoses: Revisão de Literatura. Veterinária e Zootecnia 2009; 16(4):601-616.

15. Mata-Santos T, Pinto NF, Mata-Santos HA, De Moura KG, Carneiro PF, Carvalho TS, Del Rio KP, Pinto MCFR, Martins LR, Fenalti JM, Da Silva PEA, Scaini CJ. Anthelmintic activity of lapachol, $\beta$-lapachone and its derivatives against Toxocara canis larvae. Rev Inst Med Trop Sao Paulo 2015; 57(3):197-204. 
16. Lechopier N. Quatro tensões na Saúde Pública. Est Av 2015; 29(83):209-231.

17. Winslow CEA. The untilled fields of public health. Science 1920; 51(1306):22-33.

18. Institute of Medicine. The future of public health. Washington: National Academy Press [serial on the internet]; 1988. [cited 2016 Mai 15]. Available from: http:// www.nap.edu/read/1091/chapter/1

19. Norvick LF, Morrow CB, Mays GP. Public health administration: principles for population-based management, Jones and Bartlett publisher. [serial on the internet] 2008 [cited 2016 mai]. Available from: file:///C:/ Users/Usuario/Downloads/JONES\%20AND\%20 BARTLETT\%20\%20PUBLISHERS.\%20FALA $\% 20$ DE\%20WINSLOW\%201920.\%20\%20CAPA.pdf

20. Costa JSD, Victora CG. O que é "um problema de Saúde Pública"? Rev Bras Epidemiol 2006; 9(1):144-151.

21. Araújo FR, Avalhaes J, Miyoshi MI, Salgado FB, Silva MA, Pereira ML. Contaminação de praças públicas de Campo Grande, Mato Grosso do Sul, Brasil, por ovos de Toxocara e Ancylostoma em fezes de cães. Rev Soc Bras Med Trop 1999; 32(5):581-583.

22. Andrade LD. Aspectos clínico epidemiológicos da toxocaríase humana. Revista de Patologia Tropical, 2000; 29(2):147-159.

23. Mundim TCD, Oliveira Júnior SD, Rodrigues DC, Cury MC. Frequência de helmintos em gatos de Uberlândia, Minas Gerais. Arq Bras Med Vet Zootec 2004; 56(4):562563.

24. Teixeira CR, Chieffi PP, Lescano SAZ, Silva EOM, Fux B, Cury MC. Frequency and risk factors for toxocariasis in children from a pediatric outpatient center in southeastern Brazil. Rev Inst Med Trop 2006; 48(5):251255.

25. Souza FD, Mamede-Nascimento TL, Santos CS. Encontro de ovos e larvas de helmintos no solo de praças públicas na zona sul da cidade do Rio de Janeiro. Revista de Patologia Tropical 2007; 36(3):247-253.

26. Marques JP, Guimarães CR, Vilas Boas A, Carnaúba PU, Moraes J. Contamination of public parks and squares from Guarulhos (São Paulo State, Brazil) by Toxocara spp. and Ancylostoma spp. Rev Inst Med Trop Sao Paulo 2012; 54(5):267-271.

27. Moura MQ, Jeske S, Vieira JN, Corrêa TG, Berne MEA, Villela MM. Frequency of geohelminths in public squares in Pelotas, RS, Brazil. Rev Bras Parasitol Vet 2013; 22(1):175-178.

28. Ramos DGS, Scheremeta RGAC, Oliveira ACS, Sinkoc AL, Pacheco RC. Survey of helminth parasites of cats from the metropolitan area of Cuiabá, Mato Grosso, Brazil. Rev Bras Parasitol Vet 2013; 22(2):201-206.

29. Stalliviere FM, Rosa LD, Bellato V, Souza AP, Sartor AA, Moura AB. Helmintos intestinais em cães domiciliados e aspectos socioeconômicos e culturais das famílias proprietárias dos animais de Lages, SC, Brasil. Arc Vet Science 2013; 18(3):22-27.

30. Sprenger KL, Green KT, Molento MB. Geohelminth of public areas in Curitiba, Brazil. Braz J Vet Parasitol 2014; 23(1)69-73.

31. Magoti LP. Padronização de técnica para recuperação de ovos de Toxocara canis em solo [dissertação]. São Paulo: Universidade do oeste paulista; 2008.
32. Santarém VA, Rubsnsky-Elefant G, Chesine PAF, Leli FNC. Toxocaríase canina e humana. Veterinária e Zootecnia 2009; 16(3):437-447.

33. Cassenote AJF, Neto JMP, Lima-Catelani ARA, Ferreira AW. Contaminação do solo por geo-helmintos na Cidade de Fernandópolis-SP. Rev Soc Bras Med Trop 2011; 44(3):371-374.

34. Souza RF, Dattoli VCC, Mendonça LR, Jesus JR, Santana TB, Carvalho C, Santos NM, Barrouin-Melo SM, Alcantara-Neves NM. Prevalência e fatores de risco da infecção humana por Toxocara canis em Salvador, Estado da Bahia R Rev Soc Bras Med Trop 2011; 44(4):516-519.

35. Rocha S, Pinto RMF, Floriano AP, Teixeira LH, Bassili B, Martinez A, Costa SOP, Caseiro MM. Environmental analyses of the parasitic profile found in the sandy soil from the Santos municipality beaches, SP, Brazil. Rev Inst Med Trop Sao Paulo 2011; 53(5):277-281.

36. Chen AA, Mucci JLN. Frequência de contaminação por helminto sem área de recreação infantil de creches no Município de Várzea Paulista, São Paulo, Brasil. Revista de Patologia Tropical 2012; 41(2):195-202.

37. Leavell HR. Planejamento para a Saúde Comunitária. In: Leavell HR, Clark EG, editors. Medicina Preventiva. São Paulo: MacGraw Hill do Brasil; 1976.

38. Clark DW, Macmahon B. Preventive Medicine. Boston: Little Brown and Company; 1967.

39. Morley D. Pediatria no mundo em desenvolvimento: prioridades. São Paulo: Edições Paulinas; 1980.

40. Fowler G, Austoker J. Screening. In: Detels R, Holland WW, McEwen J, Omenn GS, editors. Oxford Textbook of Public Health. New York: Oxford University Press; 1997. p. 1583-1599.

41. Daly B, Watt R, Batchelor P, Treasure E. Essential Dental Public Health. New York: Oxford Press University; 2002.

42. Capuano DM, Rocha GM. Environmental contamination by Toxocara sp. eggs in Ribeirão Preto, São Paulo State, Brazil. Rev Inst Med Trop Sao Paulo 2005; 47(4)223-226.

43. Lescano SZ, Chieffi PP, Neto AV, Ikai DK, Ribeiro MCS. A. Anti-helmínticos na toxocaríase experimental: efeito na recuperação de larvas de Toxocara canis e na resposta humoral. J Bras Patol Med Lab 2005; 41(1):21-24.

44. Lima JL, Andrade LD, Aguiar-Santos AM, Alves LC, Medeiros Z. Contaminação por ovos de Toxocara sp. em solo no município de Moreno, Estado de Pernambuco, Brasil. Braz J Vet Res Anim Sci 2005; 42(5):339346.

45. Oliveira VSF, Melo DPG, Fernandes PR, Schulze CMB, Guimarães MS, Silva AC. Ocorrência de helmintos gastrintestinais em cães errantes na cidade de Goiânia Goiás. Revista de Parasitologia Tropical 2009; 38(4):279283.

46. Santarém VA, Magoti LP, Sichieri TD. Influence of variables on centrifuge-flotation technique for recovery of Toxocara canis eggs from soil. Rev Inst Med Trop Sao Paulo 2009; 51(3):163-167.

47. Cassenote AJF. Frequência de anticorpos anti-Toxocara spp. em escolares do município de Fernandópolis-SP, Brasil e análise da contaminação do solo por ovos do parasito [dissertação]. São Paulo: Universidade de São Paulo; 2010. 
48. Gallina T, Silva MAMP, Castro LLD, Wendt LW, Villela MM, Berne MEA. Presence of eggs of Toxocara spp. and hookworms in a student environment in Rio Grande do Sul, Brazil. Rev. Bras. Parasitol. Vet. 2011; 20(2):176-177.

49. Regis SCS, Mendonça LR, Silva NS, Dattoli VCC, Alcântara-Neves NM, Barrouin-MELO S. Seroprevalence and risk factors for canine toxocariasis by detection of specific IgG as a marker of infection in dogs from Salvador, Brazil. Acta Tropica, 2011; 120(1-2):46-51.

50. Santarém VA, Leli FNC, Rubinsky-Elefant G, Giuffrida R. Protective and risk factors for toxocariasis in children from two different social classes of Brazil. Rev Inst Med Trop Sao Paulo 2011; 53(2):67-72.

51. Mendonça LR, Figueiredo CA, Esquivel R, Fiaccone RL, Pontes-de-Carvalho CP, Barreto ML, Alcantara-Neves $\mathrm{MN}$. Seroprevalence and risk factors for Toxocara infection in children from an urban large setting in Northeast Brazil. Acta Trop 2013; 128(1)90-95.

52. Ribeiro LM, Dracz RM, Mozzer LR, Lima WS. Soil contamination in public squares in Belo Horizonte, Minas Gerais, by canine parasites in different developmental stages. Rev Inst Med Trop Sao Paulo 2013; 55(4):229231.

53. Salamaia FH, Lopes RC, Molinari-Capel LM. Estudo de parasitas intestinais caninos provenientes de cães hospedados no canil e escola Emanuel, Maringá-PR. Rev Saúde Pública 2013; 6(1):27-33.

54. Onuma SSM, Melo ALT, Stocco MB, Santarém VA, Aguiar DM. Contaminação de solo por ovos de Toxocara spp. e outros geo-helmintos em comunidade rural do Pantanal Mato-Grossense, Brasil. Braz J Vet Res Anim. Sci, 2014; 51(1):78-81.

55. Barra LAC, Santos WF, Chieffi PP, Bedaque EA, Campos Salles PS, Capitão CG, Vianna S, Hanna R, Pedretti Júnior L. Larva migrans visceral: forma mista de apresentação em adulto. Aspectos clínicos e laboratoriais. Rev Inst Med Trop Sao Paulo 1996; 29(4):373-376.

56. Silva HC, Castagnolli C, Silveira DM, Costa GHN, Gomes RA, Nascimento AA. Fauna helmíntica de cães e gatos provenientes de alguns municípios do Estado de São Paulo. Semina: Ci. Agrárias, Londrina 200; 22(1):67-71.

57. Alderete JM, Jacob CMA, Pastorino AC, Elefant GR, Castro APM, Fomin ABF, Chieffi PP. Prevalence of Toxocara infection in schoolchildren from the Butantã region, Sao Paulo, Brazil. Mem Inst Oswaldo Cruz 2003; 98(5)593-597.

58. Moreira-Silva SF, Rodrigues MG, Pimenta JL, Gomes CP, Freire LH, Pereira FEL. Toxocariasis of the central nervous system: with report of two cases. Rev Inst Med Trop Sao Paulo 2004; 37(2)169-174.

59. Coêlho RAL, Carvalho Júnior LB, Perez EP, Araki K, Takeuchi T, Ito A, Aoki T, Yamasaki H. Prevalence of Toxocariasis in Northeastern Brazil based on serology using recombinant Toxocara canis antigen. Am J Trop Med Hyg 2005; 72(1):103-107.

60. Guimarães AM, Alves EGL, Rezende GF, Rodrigues MC. Ovos de Toxocara sp. e larvas de Ancylostoma sp. em praça pública de Lavras, MG. Rev Saúde Pública 2005; 32(2):293-295.
61. Queiroz ML, Simonsen M, Paschoalotti MA, Chieffi PP. Frequency of soil contamination by Toxocara canis eggs in the South Region of São Paulo municipality (SP, Brazil) in a 18 month period. Rev Inst Med Trop Sao Paulo 2006; 48(60):317-319.

62. Lima AMA, Alves LC, Faustino MAG, Lira MAS, Magalhães A, Lima MM, Teixeira WC, Borges JCG, Pimentel DS. Búsqueda de huevos de anquilostomideos y toxocarídeos en el suelo de residencias y escuelas en el barrio de dois irmãos, Recife-PE (Brasil). Parasitol Latinoam 2007; 62(1-2)89-93.

63. Santarém VA, Franco EC, Kozuki FT, Fini D, PrestesCarneiro L. Environmental contamination by Toxocara spp. eggs in a rural settlement in Brazil. Rev Inst Med Trop Sao Paulo 2008; 50(5):279-281.

64. Teixeira ML, Rossi LP, Freitas L, Gasparin N, Piva S, Fuentefria AM. Prevalence of Toxocara canis infection in public squares of the Concórdia City, Santa Catarina. Brazil. Parasitol Latinoam 2008; 63(1-2)69-71.

65. Chieffi PP, Santos SV, Queiroz ML, Lescano SAZ. Human toxocariasis: contribution by Brazilian researchers. Rev Inst Med Trop Sao Paulo 2009; 51(6):301-308.

66. Frassy LN, Braga FR, Silva AR, Araújo JV, Ferreira SR, Freitas LG. Destruição de ovos de Toxocara canis pelo fungo nematófogo Pochonia chlamydosporia. Rev Soc Bras Med Trop 2010; 43(1):102-104.

67. Ostermann AM. Infecção por helmintos gastrintestinais em cães (Canis familiaris, Linnaeus, 1758) errantes procedentes do município de Recife-PE. [Tese]. Recife: Universidade Federal Rural de Pernambuco; 2008.

68. Figueiredo MIO, Wendt EW, Santos H, Moreira CM. Levanta mento sazonal de parasito sem caixas de areia nas escolas municipais de educação infantil em Uruguaiana, RS, Brasil. Revista de Patologia Tropical 2012; 41(1):36-46.

69. Matesco VC, Rott MB, Mentz MB. Comparação entre métodos de centrífugo flutuação utilizados para a recuperação de ovos de helmintos em amostras de areia. Revista de Patologia Tropical 2011; 40(4):323-330.

70. Ostermann AM, Lima MM, Farias MPO, D'alencar AS, Galindo MKF, Silva CT, Alves LC, Faustino MAG. Comparação entre exames coproparasitológicos e necroscópicos para diagnóstico da infecção por helmintos gastrintestinais em cães (Canis familiaris, Linnaeus, 1758) errantes provenientes da Região Metropolitana do Recife-PE. Revista Biotemas 2011; 24(2):47-55.

71. Fisher M. Toxocara cati: an underestimated zoonotic agent. Trends Parasitol 2003; 19(4):167-170.

72. Bresciani KDS, Ishizaki MN, Kaneto CN, Montano TRP, Perri SHV, Vasconcelos R. O, Do Nascimento AA. Frequência e intensidade parasitária de helmintos gastrintestinais em cães na área urbana do Município de Araçatuba, SP. ARS Veterinaria, 2008; 24(3):181-185.

73. Moreira-Silva SF, Leão ME, Mendonça HF, Pereira FE. Prevalence of anti-Toxocara antibodies in a random sample of inpatients at a chidren's hospital in Vitória, Espirito Santo, Brazil. Rev Inst Med Trop Sao Paulo 1998; 40(4);259-261.

74. Anaruma FF, Chieffi PP, Correia CRS, Camargo ED, Silveira EPR, Aranha JJB. Human toxocariasis: incidence among residents in the outskirts of Campinas, São Paulo, Brazil. Rev Inst Med Trop Sao Paulo 2003; 45(5):293-294. 
75. Paludo LM, Falavigna DLM, Rubinski-Elefant G, Gomes LG, Baggio MLM, Amadei LB, Falavigna-Guilherme AL. Frequency of Toxocara infection in children attended by the health public service of Maringá, South Brazil. Rev Inst Med Trop Sao Paulo 2007; 49(1):343348.

76. Colli CM, Rubinsky-Elefant G, Paludo ML, Falavigna DLM, Guilherme EV, Mattia S, Araújo SM, Ferreira EC, Previdelli ITS, Falavigna-Guilherme AL. Serological, clinical and epidemiological evaluation of toxocariasis in urban areas of south Brazil. Rev Inst Med Trop Sao Paulo 2010; 52(2):69-74.

77. Engbaek K, Madsen H, Larsen SO, A survey of helminths in stray cats Copenhagen with ecological aspects. Z Parasitenkd 1984; 70(1):87-94.

78. Cáceres APG, Gonçalves FA, Cazorla IM, Carvalho SMS. Contaminação do solo por helmintos de importância médica na praia do Sul (Milionários), Ilhéus BA. News Lab 2004; 67(1):146-155.

79. Blazius RD, Silva OS, Kauling AL, Rodrigues DFP, Lima MC. Contaminação da areia do Balneário de Laguna, SC, por Ancylostoma spp., e Toxocara spp., em amostras fecais de cães e gatos. Arquivos Catarinenses de Medicina 2006; 35(3)55-58.

80. Nunes CM, Pena FC, Negrelli GB, Anjo CGS, Nakano MM, Stobbe NS. Ocorrência de larva migrans na areia de áreas de lazer das escolas municipais de ensino infantil, Araçatuba, SP, Brasil. Rev Saude Publica 2000; 34(6):656-658.

81. Oliveira CAF, Germano PML. Estudo da ocorrência de enteroparasitas em hortaliças comercializadas na Região Metropolitana de São Paulo-SP, Brasil. Pesquisa de helmitos. Rev Saúde Pública 1992; 26(4):283-289.

82. Guilherme ALF, Araújo SM, Falavigna DLM, Pupulim ART, Dias MLGG, Oliveia HS, Maroco E, Yoshiaki F. Prevalência de enteroparasitos em horticultores e hortaliças da feira do produtor de Maringá, Paraná. Rev Soc Bras Med Trop 1999; 32(4):405-411.

83. Ferro JJB, Costa-Cruz JM, Barcelos IS. Avaliação parasitológica de alfaces (lactuca sativa) comercializadas no município de Tangará da Serra, Mato Grosso, Brasil. Revista de Patologia Tropical, 2012; 41(1):47-54.

84. Nunes CM, Tundisi RN, García JF. Cross reactions between Toxocara canis and Ascaris suum in the diagnosis of visceral larva migrans by western blotting technique. Rev Inst Med Trop Sao Paulo 1997; 39(5):253-256.

85. Moreira-Silva SF, Leão ME, Mendonça HFS, Pereira FEL. Prevalence of anti-Toxocara antibodies in a random sample of inpatients at a children's hospital in Vitória, Espírito Santo, Brazil. Rev Inst Med Trop Sao Paulo 1998; 40(5):259-261.

Artigo apresentado em 19/07/2016

Aprovado em 14/01/2017

Versão final apresentada em 16/01/2017 